\title{
Q (Q Comparative risk of major congenital malformations with eight different antiepileptic drugs: a prospective cohort study of the EURAP registry
}

\author{
Torbjörn Tomson*, Dina Battino*, Erminio Bonizzoni, John Craig, Dick Lindhout, Emilio Perucca, Anne Sabers, Sanjeev V Thomas, Frank Vajda, \\ for the EURAP Study Group $†$
}

\section{Summary}

Lancet Neurol 2018; 17: 530-38

Published Online

April 18, 2018

http://dx.doi.org/10.1016/

S1474-4422(18)30107-8

See Comment page 485

*Contributed equally

†The appendix shows the

complete list of collaborators

Department of Clinical

Neuroscience, Karolinska

Institutet, Stockholm, Sweden

(ProfTTomson MD);

Department of Neurology,

Karolinska University Hospital,

Stockholm, Sweden

(ProfTTomson); Epilepsy

Center, Department of

Neurophysiology and

Experimental Epileptology,

IRCCS Neurological Institute

Carlo Besta Foundation, Milan,

Italy (D Battino MD);

Department of Clinical Science

and Community, Section of

Medical Statistics, Biometry

and Epidemiology, Faculty of

Medicine and Surgery,

University of Milan, Milan, Italy

(E Bonizzoni PhD); Department

of Neurosciences, Acute and

Unscheduled Care, Belfast

Health and Social Care Trust,

Belfast, UK (J Craig MB);

Department of Genetics,

University Medical Center

Utrecht, Utrecht, Netherlands

(Prof D Lindhout MD); Stichting

Epilepsie Instellingen

Nederland (SEIN), Heemstede,

Netherlands (Prof D Lindhout);

Department of Internal

Medicine and Therapeutics,

University of Pavia, Pavia, Italy

(Prof E Perucca MD); Clinical

Trial Center, IRCCS Mondino

Foundation, Pavia, Italy

(Prof E Perucca); The Epilepsy Clinic, Department of

Neurology, Rigshospitalet-

Blegdamsvej, University State

Hospital, Copenhagen,

Denmark (Prof A Sabers MD);

Department of Neurology, Sree

ChitraTirunal Institute of

Medical Sciences and

Technology, Trivandrum,

Background Evidence for the comparative teratogenic risk of antiepileptic drugs is insufficient, particularly in relation to the dosage used. Therefore, we aimed to compare the occurrence of major congenital malformations following prenatal exposure to the eight most commonly used antiepileptic drugs in monotherapy.

Methods We did a longitudinal, prospective cohort study based on the EURAP international registry. We included data from pregnancies in women who were exposed to antiepileptic drug monotherapy at conception, prospectively identified from 42 countries contributing to EURAP. Follow-up data were obtained after each trimester, at birth, and 1 year after birth. The primary objective was to compare the risk of major congenital malformations assessed at 1 year after birth in offspring exposed prenatally to one of eight commonly used antiepileptic drugs (carbamazepine, lamotrigine, levetiracetam, oxcarbazepine, phenobarbital, phenytoin, topiramate, and valproate) and, whenever a dose dependency was identified, to compare the risks at different dose ranges. Logistic regression was used to make direct comparisons between treatments after adjustment for potential confounders and prognostic factors.

Findings Between June 20, 1999, and May 20, 2016, 7555 prospective pregnancies met the eligibility criteria. Of those eligible, 7355 pregnancies were exposed to one of the eight antiepileptic drugs for which the prevalence of major congenital malformations was 142 (10.3\%) of 1381 pregnancies for valproate, $19(6 \cdot 5 \%)$ of 294 for phenobarbital, eight (6.4\%) of 125 for phenytoin, 107 (5.5\%) of 1957 for carbamazepine, six (3.9\%) of 152 for topiramate, ten (3.0\%) of 333 for oxcarbazepine, $74(2.9 \%)$ of 2514 for lamotrigine, and $17(2.8 \%)$ of 599 for levetiracetam. The prevalence of major congenital malformations increased with the dose at time of conception for carbamazepine $(p=0.0140)$, lamotrigine $(p=0.0145)$, phenobarbital $(p=0 \cdot 0390)$, and valproate $(p<0 \cdot 0001)$. After adjustment, multivariable analysis showed that the prevalence of major congenital malformations was significantly higher for all doses of carbamazepine and valproate as well as for phenobarbital at doses of more than $80 \mathrm{mg} / \mathrm{day}$ than for lamotrigine at doses of $325 \mathrm{mg} /$ day or less. Valproate at doses of $650 \mathrm{mg} /$ day or less was also associated with increased risk of major congenital malformations compared with levetiracetam at doses of 250-4000 mg/day (odds ratio [OR] 2.43, 95\% CI 1.30-4.55; p=0.0069). Carbamazepine at doses of more than $700 \mathrm{mg} / \mathrm{day}$ was associated with increased risk of major congenital malformations compared with levetiracetam at doses of 250-4000 mg/day (OR 2.41, 95\% CI 1.33-4.38; p=0.0055) and oxcarbazepine at doses of 75-4500 mg/day $(2 \cdot 37,1 \cdot 17-4 \cdot 80 ; \mathrm{p}=0 \cdot 0169)$.

Interpretation Different antiepileptic drugs and dosages have different teratogenic risks. Risks of major congenital malformation associated with lamotrigine, levetiracetam, and oxcarbazepine were within the range reported in the literature for offspring unexposed to antiepileptic drugs. These findings facilitate rational selection of these drugs, taking into account comparative risks associated with treatment alternatives. Data for topiramate and phenytoin should be interpreted cautiously because of the small number of exposures in this study.

Funding Bial, Eisai, GlaxoSmithKline, Janssen-Cilag, Novartis, Pfizer, Sanofi-Aventis, UCB, the Netherlands Epilepsy Foundation, and Stockholm County Council.

Copyright (C) 2018 Elsevier Ltd. All rights reserved.

\section{Introduction}

The teratogenic risks associated with antiepileptic drugs are a concern for prescribers from many disciplines because epilepsy is often managed by primary care physicians and there has been an increasing use of these drugs for indications other than epilepsy. Evidence suggests that infants exposed to antiepileptic drugs are at higher risk for major congenital malformations than are infants of untreated mothers with epilepsy. ${ }^{1}$ Observational studies have reported that valproate has a higher teratogenic risk than other antiepileptic drugs, ${ }^{2-7}$ that this risk is dose dependent, ${ }^{2,3,5,7}$ and that offspring exposed prenatally to valproate are also at higher risk for impaired postnatal cognitive development ${ }^{8-10}$ and, possibly, autism 


\section{Research in context}

\section{Evidence before this study}

Although the use of antiepileptic drugs during pregnancy has been known for more than 50 years to be associated with increased risks of major congenital malformations in the offspring, it is not until more recent years, with emerging data from prospective pregnancy registries, that the teratogenic potential of different antiepileptic drugs had begun to be delineated. We refer to two recently published systematic reviews and meta-analyses. One review focused on newer antiepileptic drugs and searched, on Nov 12, 2014, PubMed and Embase for all newer antiepileptic drugs, congenital anomalies, and pregnancy. The second systematic review searched MEDLINE, Embase, and the Cochrane Central Register of Controlled trials from inception to Dec 15, 2015. We did a separate PubMed search for articles published from Dec 16, 2015, to Dec 15, 2017, using the search terms "anticonvulsants", "antiepileptic drugs", "teratogenicity", and "birth defects". Our search was limited to studies in English and we did not set any criteria for assessment of quality.

Although meta-analyses increase the statistical power, they combine data from heterogeneous studies sometimes with different outcome criteria, and do not provide direct within-study comparisons between antiepileptic drug treatments. Such internal comparisons have been made in individual reports from independent prospective antiepileptic drug and pregnancy registries but so far only between the most frequently used antiepileptic drugs. Taken together, these studies have consistently reported increased risk of major congenital malformations associated with valproate and phenobarbital compared with pregnancies unexposed to antiepileptic drugs, as well as compared with pregnancies exposed to other antiepileptic drugs such as carbamazepine and lamotrigine. Findings also suggest increased risks associated with topiramate. Pregnancy registries have also reported that the risks associated with some drugs, in particular valproate, might be dose dependent.

\section{Added value of this study}

On the basis of the largest published prospective cohort of pregnancies in women with epilepsy so far, we provide the prevalence of major congenital malformations at 1 year after birth following exposure to the eight most frequently used antiepileptic drugs in monotherapy. Where a dose dependency relationship was apparent, risks are reported at different doses used at conception. In a multivariable analysis, we report 36 direct comparisons of risks between different treatment modalities.

\section{Implications of all the available evidence}

With epilepsy often managed by primary care physicians, and an increasing use of antiepileptic drugs for other indications, the risk of major congenital malformations is a concern for prescribers from many disciplines. Epilepsy, as well as some psychiatric conditions, might have serious consequences and require continuation of treatment during pregnancy. Treatment modalities should be reviewed regularly in all women of childbearing potential to assess risks and benefits of treatment alternatives and enable any appropriate modifications before pregnancy. Current evidence has made clear that, whenever possible, valproate should be avoided in the treatment of women of childbearing potential, but risks differ also between other treatments. Our comprehensive study, with comparisons of risks between a multitude of antiepileptic drug treatments and dosages, provides physicians with vital information when they consider alternatives for women of childbearing potential. spectrum disorders. ${ }^{11}$ On the basis of these observations, the US Food and Drug Administration ${ }^{12}$ and the European Medicines Agency ${ }^{13}$ issued restrictions on the use of valproate in women of childbearing age and, in the case of the European Medicines Agency, also in girls.

The use of valproate for the treatment of epilepsy in pregnancy ${ }^{14}$ and in women of childbearing age has recently declined. ${ }^{15}$ By contrast, the use of valproate for other indications has increased, ${ }^{15}$ in particular for psychiatric conditions, ${ }^{14}$ which might reflect a gap in awareness among those outside the field of neurology. ${ }^{14}$ Prescription patterns, however, might also reflect the shortage of appropriate treatment alternatives and insufficient data for the teratogenicity of other antiepileptic drugs, ${ }^{16}$ particularly newer generation antiepileptic drugs, the use of which has increased substantially in pregnancy. ${ }^{14,17}$

Information about comparative risks of major congenital malformations with different antiepileptic drugs comes primarily from prospective registries..$^{2-5,18}$ However, because of power limitations, so far only the most frequently used antiepileptic drugs have been assessed.
Meta-analyses might increase statistical power, but are limited by methodological heterogeneity across included studies and fail to assess important variables such as the influence of the dose used. ${ }^{1,19}$ Therefore to overcome these limitations, we aimed to compare the risks of major congenital malformations for eight major antiepileptic drugs, including the risks associated with different doses of the most commonly used agents to provide physicians with novel tools for rational selection of these drugs.

\section{Methods}

\section{Study design and participants}

We did a longitudinal prospective, cohort study based on EURAP, an international registry collecting data for the assessment of risks of antiepileptic drugs during pregnancy. EURAP was established in 1999 and has since involved 42 countries with more than 1500 collaborators (appendix).

We included data for pregnant women for prospective assessment if they were exposed to antiepileptic drugs at conception, enrolled in EURAP within gestation week 16
Kerala, India

(Prof SVThomas MD); and Department of Medicine and Department of Neurology, University of Melbourne, Royal Melbourne Hospital, Melbourne, VIC, Australia (Prod F Vajda MD)

Correspondence to: ProfTorbjörn Tomson Department of Neurology, Karolinska University Hospital, SE 17176 Stockholm, Sweden torbjorn.tomson@sll.se

See Online for appendix 
(based on the date of the last menstrual period), and fetal outcome was unknown. We excluded from the current analysis retrospectively identified pregnancies, those occurring in women without epilepsy, those for which physicians did not submit reports within preset deadlines, and those for which follow-up was not yet completed at the current census. We also excluded pregnancies in which antiepileptic drugs were switched

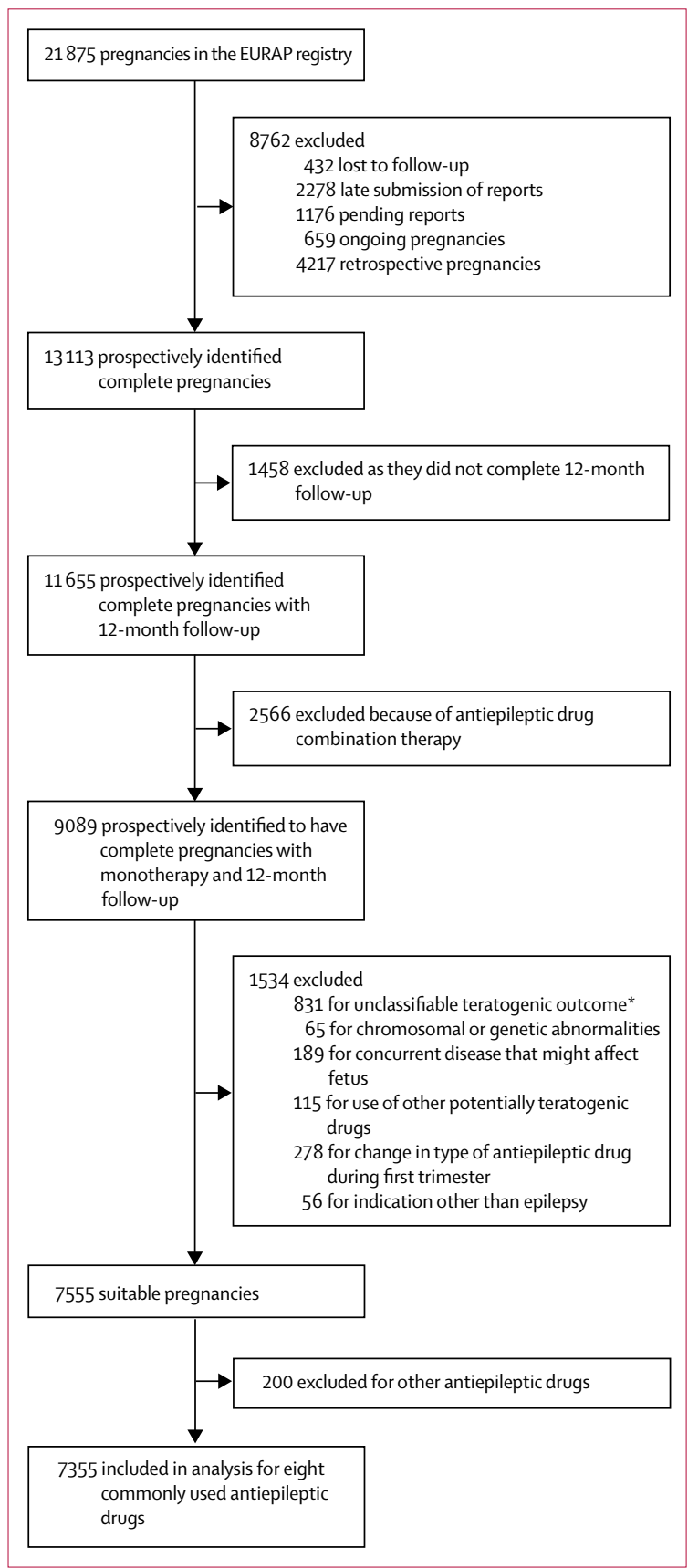

Figure: Study population and selection process

*Unclear whether there was a major malformation $(n=27)$, intrauterine deaths ( $n=693)$, or induced abortions for causes other than fetal abnormalities $(n=111)$.

or withdrawn during the first trimester, those exposed to antiepileptic drug combination therapy or to other potentially teratogenic drugs, and those with comorbidities associated with teratogenic risks (appendix). Additionally, we excluded cases of 
spontaneous abortions, abortions induced for causes other than fetal abnormalities, pregnancies in which fetal outcome could not be determined, and pregnancies that resulted in offspring with genetic or chromosomal abnormalities (appendix).

We received approval from the ethics committees of participating centres for the protocol, and we obtained either written or oral informed consent from all women enrolled.

\section{Procedures}

We obtained information during early pregnancy about demographics, type of epilepsy, seizure frequency, comorbidities, family history of major congenital malformations, type of antiepileptic drug treatment used, and other risk factors. Additionally, we obtained follow-up data after each trimester, at birth, and 1 year after birth. The treating physician collected these data and transferred it online to a EURAP national coordinator, who reviewed the reports for completeness and accuracy before transmission to the EURAP central database in Milan, Italy.

Abnormalities in the offspring were reported descriptively by enrolling physicians, who were instructed to report anything they considered to be a potential abnormality. These reports were reviewed and classified by an outcome committee unaware of the type of exposure. When needed, the committee could request supplementary information from the reporting physician.

\section{Outcomes}

The primary objective was to compare the risk of major congenital malformations assessed at 1 year after birth in offspring exposed prenatally to one of eight different antiepileptic monotherapies (carbamazepine, lamotrigine, levetiracetam, oxcarbazepine, phenobarbital, phenytoin, topiramate, and valproate) and, whenever a dose dependency was identified, to compare the risks at different dose ranges.

Pregnancies were classified on the basis of type and dosage of antiepileptic drug used at conception. Seizures were classified as either generalised tonic-clonic or other types, and epilepsy syndromes broadly classified according to the International League Against Epilepsy criteria at the time EURAP was launched. ${ }^{20}$ Major congenital malformations were defined as structural abnormalities with surgical, medical, functional, or cosmetic importance, and classified according to the 2005 EUROCAT criteria. ${ }^{21}$

Covariates for the analysis were selected a priori on the basis of those considered potentially relevant based on our previous analysis of pregnancy outcome in EURAP. Family history of major congenital malformations refers to the proband's parents, excluding cerebral malformations causally associated with the maternal epilepsy. Folate supplementation was arbitrarily deemed appropriate if initiated at least 3 months before conception and maintained throughout the first trimester irrespective of dose, provided that it was no less than $0.4 \mathrm{mg} /$ day.

\begin{tabular}{|lclll}
\hline & $\begin{array}{l}\text { Dose range } \\
\text { (mg/day) }\end{array}$ & $\begin{array}{l}\text { Number of } \\
\text { pregnancies } \\
\text { exposed }\end{array}$ & $\begin{array}{l}\text { Number of major } \\
\text { congenital } \\
\text { malformation events }\end{array}$ & $\begin{array}{l}\text { Prevalence of major } \\
\text { congenital malformation } \\
\text { events }(95 \% \text { Cl) }\end{array}$ \\
\hline Lamotrigine & $25-1300$ & 2514 & 74 & $2 \cdot 9 \%(2 \cdot 3-3 \cdot 7)$ \\
\hline Carbamazepine & $50-2400$ & 1957 & 107 & $5 \cdot 5 \%(4 \cdot 5-6 \cdot 6)$ \\
\hline Valproate & $100-3000$ & 1381 & 142 & $10 \cdot 3 \%(8 \cdot 8-12 \cdot 0)$ \\
\hline Levetiracetam & $250-4000$ & 599 & 17 & $2 \cdot 8 \%(1 \cdot 7-4 \cdot 5)$ \\
Oxcarbazepine & $75-4500$ & 333 & 10 & $3 \cdot 0 \%(1 \cdot 4-5 \cdot 4)$ \\
\hline Phenobarbital & $15-300$ & 294 & 19 & $6 \cdot 5 \%(4 \cdot 2-9 \cdot 9)$ \\
\hline Topiramate & $25-500$ & 152 & 6 & $3 \cdot 9 \%(1 \cdot 5-8 \cdot 4)$ \\
\hline Phenytoin & $30-730$ & 125 & 8 & $6 \cdot 4 \%(2 \cdot 8-12 \cdot 2)$ \\
\hline
\end{tabular}

Table 2: Prevalence of major congenital malformations in offspring exposed prenatally to one of eight different antiepileptic monotherapies

\begin{tabular}{|c|c|c|c|c|}
\hline & $\begin{array}{l}\text { Number of } \\
\text { pregnancies } \\
\text { exposed }\end{array}$ & $\begin{array}{l}\text { Number of major } \\
\text { congenital } \\
\text { malformation events }\end{array}$ & $\begin{array}{l}\text { Prevalence of major } \\
\text { congenital malformation } \\
\text { events }(95 \% \mathrm{Cl})\end{array}$ & $p$ value \\
\hline \multicolumn{5}{|l|}{ Lamotrigine } \\
\hline$\leq 325 \mathrm{mg} /$ day & 1870 & 46 & $2 \cdot 5 \%(1 \cdot 8-3 \cdot 3)$ & 0.0145 \\
\hline$>325$ mg/day & 644 & 28 & $4 \cdot 3 \%(2 \cdot 9-6 \cdot 2)$ & .. \\
\hline \multicolumn{5}{|l|}{ Carbamazepine } \\
\hline$\leq 700 \mathrm{mg} /$ day & 1276 & 58 & $4 \cdot 5 \%(3 \cdot 5-5 \cdot 8)$ & 0.0140 \\
\hline$>700$ mg/day & 681 & 49 & $7 \cdot 2 \%(5 \cdot 4-9 \cdot 4)$ & .. \\
\hline \multicolumn{5}{|l|}{ Valproate } \\
\hline$\leq 650 \mathrm{mg} /$ day & 600 & 38 & $6 \cdot 3 \%(4 \cdot 5-8 \cdot 6)$ & $<0.0001$ \\
\hline$>650$ to $\leq 1450 \mathrm{mg} /$ day & 666 & 75 & $11 \cdot 3 \%(9 \cdot 0-13 \cdot 9)$ & .. \\
\hline$>1450$ mg/day & 115 & 29 & $25 \cdot 2 \%(17 \cdot 6-34 \cdot 2)$ & .. \\
\hline \multicolumn{5}{|l|}{ Phenobarbital } \\
\hline$\leq 80 \mathrm{mg} /$ day & 73 & 2 & $2 \cdot 7 \%(0 \cdot 3-9 \cdot 5)$ & 0.0390 \\
\hline$>80$ to $\leq 130 \mathrm{mg} /$ day & 161 & 10 & $6 \cdot 2 \%(3 \cdot 0-11 \cdot 1)$ & .. \\
\hline$>130$ mg/day & 60 & 7 & $11 \cdot 7 \%(4 \cdot 8-22 \cdot 6)$ & .. \\
\hline
\end{tabular}

When a dose dependency for the risk of major congenital malformation was identified, comparisons also included specific dose ranges at time of conception.

Table 3: Association between prevalence of major congenital malformations and exposure to one of the four monotherapies in which a dose response was detectable

\section{Statistical analysis}

A total sample of 7000 observations achieves $80 \%$ power at a two-sided 0.05 significance level to detect an odds ratio $(\mathrm{OR})$ of $2 \cdot 0$, based on logistic regression of a binary response variable on a binary covariate with a prevalence ranging from $10 \%$ to $90 \%$ and a $2 \%$ frequency of teratogenic events in the group with the lowest frequency of events. On the basis of the empirical rule that the ratio between the overall number of events and the number of explanatory variables should be at least equal to ten, a total sample size of 7000 observations was deemed adequate to minimise the risk of overfitting in a multivariable logistic model, assuming a 5\% frequency of teratogenic events in the pooled population and a selected number of clinically plausible predictors equal to 35 .

To calculate frequencies of major congenital malformations, the numerator was the sum of all live births 
plus pregnancy losses with confirmed major congenital malformations, whereas the denominator included live births, pregnancies terminated electively for suspected major congenital malformations, and perinatal deaths.

The EURAP dose categorisation algorithm ${ }^{5}$ was used to define dose categories. The Cochrane-Armitage test was used to confirm a dose response across the identified categories. A random effect logistic model was used to compare treatments after adjusting for potential confounders or prognostic factors, and to assess the effect of these factors on outcome. The logistic regression

\begin{tabular}{|c|c|c|}
\hline & $\begin{array}{l}\text { Odds ratio } \\
(95 \% \mathrm{Cl})\end{array}$ & $\begin{array}{l}\text { Adjusted } \\
\mathrm{p} \text { value } \dagger\end{array}$ \\
\hline \multicolumn{3}{|l|}{ Drug comparisons with lamotrigine $\leq 325 \mathrm{mg} /$ day } \\
\hline Valproate (>1450 mg/day) & $13 \cdot 52(7 \cdot 73-23 \cdot 64)$ & 0.0002 \\
\hline Phenobarbital (>130 mg/day) & $5 \cdot 81(2 \cdot 40-14 \cdot 08)$ & 0.0002 \\
\hline Valproate (>650 mg/day to $\leq 1450 \mathrm{mg} /$ day) & $4.72(3 \cdot 11-7 \cdot 18)$ & 0.0002 \\
\hline Valproate ( $\leq 650 \mathrm{mg} /$ day $)$ & $2 \cdot 70(1.67-4.38)$ & 0.0002 \\
\hline Carbamazepine (>700 mg/day) & $2.68(1.71-4 \cdot 19)$ & 0.0002 \\
\hline Phenobarbital (>80 mg/day to $\leq 130 \mathrm{mg} /$ day) & $2 \cdot 46(1 \cdot 16-5 \cdot 23)$ & 0.0196 \\
\hline Phenytoin ( $\geq 30 \mathrm{mg} /$ day to $730 \mathrm{mg} /$ day) & $1.93(0.78-4.75)$ & 0.1554 \\
\hline Carbamazepine ( $\leq 700 \mathrm{mg} /$ day $)$ & $1 \cdot 71(1 \cdot 12-2 \cdot 61)$ & 0.0143 \\
\hline Lamotrigine (>325 mg/day) & $1.68(1.01-2.80)$ & 0.0463 \\
\hline Topiramate ( $\geq 25 \mathrm{mg} /$ day to $500 \mathrm{mg} /$ day) & $1.67(0.69-4.04)$ & 0.2524 \\
\hline Oxcarbazepine ( $\geq 75 \mathrm{mg} /$ day to $4500 \mathrm{mg} /$ day) & $1.13(0.55-2 \cdot 31)$ & 0.7358 \\
\hline Levetiracetam ( $\geq 250 \mathrm{mg} /$ day to $4000 \mathrm{mg} /$ day) & $1.11(0.62-2.00)$ & $0 \cdot 7282$ \\
\hline Phenobarbital ( $\leq 80$ mg/day) & $1.07(0.25-4.60)$ & 0.923 \\
\hline \multicolumn{3}{|l|}{ Within-drug comparisons } \\
\hline Phenobarbital (>130 mg/day vs $\leq 80 \mathrm{mg} /$ day) & $5.41(1.05-27.89)$ & 0.0436 \\
\hline Valproate (>1450 mg/day vs $\leq 650 \mathrm{mg} /$ day) & $5.00(2.79-8.97)$ & 0.0002 \\
\hline Valproate (>1450 mg/day vs $>650 \mathrm{mg} /$ day to $\leq 1450 \mathrm{mg} /$ day) & $2 \cdot 86(1.67-4 \cdot 89)$ & 0.0002 \\
\hline Phenobarbital ( $>130 \mathrm{mg} /$ day $v \mathrm{~s}>80 \mathrm{mg} /$ day to $\leq 130 \mathrm{mg} /$ day) & $2.36(0.81-6.86)$ & 0.1135 \\
\hline Phenobarbital ( $>80 \mathrm{mg} /$ day to $\leq 130 \mathrm{mg} /$ day $v s \leq 80 \mathrm{mg} /$ day) & $2.29(0.47-11.05)$ & $0 \cdot 3028$ \\
\hline Valproate (>650 mg/day to $\leq 1450 \mathrm{mg} /$ day vs $\leq 650 \mathrm{mg} /$ day) & $1.75(1 \cdot 12-2 \cdot 73)$ & 0.0147 \\
\hline Lamotrigine (>325 mg/day vs $\leq 325$ mg/day) & $1.68(1.01-2 \cdot 80)$ & 0.0463 \\
\hline Carbamazepine (>700 mg/day vs $\leq 700 \mathrm{mg} /$ day) & $1.56(1.03-2 \cdot 37)$ & 0.0352 \\
\hline \multicolumn{3}{|l|}{ Other antiepileptic drug comparisons } \\
\hline Valproate (>650 mg/day to $\leq 1450 \mathrm{mg} /$ day) vs lamotrigine (>325 mg/day) & $2 \cdot 81(1 \cdot 70-4 \cdot 65)$ & 0.0002 \\
\hline $\begin{array}{l}\text { Valproate ( }>650 \mathrm{mg} / \text { day to } \leq 1450 \mathrm{mg} / \text { day }) \text { vs carbamazepine } \\
(\leq 700 \mathrm{mg} / \text { day })\end{array}$ & $2 \cdot 76(1.82-4.19)$ & 0.0002 \\
\hline Valproate ( $\leq 650 \mathrm{mg} /$ day) vs levetiracetam (250-4000 mg/day) & $2 \cdot 43(1 \cdot 30-4 \cdot 55)$ & 0.0069 \\
\hline Carbamazepine (>700 mg/day) vs levetiracetam ( $250-4000$ mg/day) & $2 \cdot 41(1 \cdot 33-4 \cdot 38)$ & 0.0055 \\
\hline Valproate ( $\leq 650$ mg/day) vs oxcarbazepine $(75-4500$ mg/day) & $2 \cdot 39(1.13-5 \cdot 08)$ & 0.0235 \\
\hline Carbamazepine (>700 mg/day) vs oxcarbazepine ( $75-4500 \mathrm{mg} /$ day) & $2 \cdot 37(1 \cdot 17-4 \cdot 80)$ & 0.0169 \\
\hline Valproate ( $\leq 650 \mathrm{mg} /$ day) vs carbamazepine ( $\leq 700 \mathrm{mg} /$ day) & $1.58(0.98-2 \cdot 55)$ & 0.0626 \\
\hline Lamotrigine (>325 mg/day) vs levetiracetam (250-4000 mg/day) & $1 \cdot 51(0 \cdot 79-2 \cdot 88)$ & $0 \cdot 2077$ \\
\hline Lamotrigine (>325 mg/day) vs oxcarbazepine (75-4500 mg/day) & $1.49(0.70-3.17)$ & $0 \cdot 3051$ \\
\hline Oxcarbazepine ( $75-4500 \mathrm{mg} /$ day) vs levetiracetam ( $250-4000 \mathrm{mg} /$ day) & $1 \cdot 02(0 \cdot 45-2 \cdot 30)$ & 0.9644 \\
\hline Carbamazepine (>700 mg/day) vs valproate $(\leq 650 \mathrm{mg} /$ day $)$ & $0.99(0.60-1.65)$ & 0.9708 \\
\hline Oxcarbazepine (75-4500 mg/day) vs carbamazepine ( $\leq 700 \mathrm{mg} /$ day) & $0.66(0.33-1 \cdot 32)$ & 0.2393 \\
\hline Levetiracetam (250-4000 mg/day) vs carbamazepine ( $\leq 700 \mathrm{mg} /$ day) & $0.65(0.36-1.16)$ & $0 \cdot 1412$ \\
\hline Lamotrigine (>325 mg/day) vs carbamazepine (>700 mg/day) & $0.63(0.38-1.05)$ & 0.0766 \\
\hline Lamotrigine (>325 mg/day) vs valproate ( $\leq 650 \mathrm{mg} /$ day) & $0.62(0.36-1.09)$ & 0.0959 \\
\hline
\end{tabular}

(Table 4 continues on next page) was done by fitting data through a generalised mixed linear model with the logit-link function, a binomial distribution, and an indicator of mother as a random effect to adjust estimates for the presence of clustered data (women with more than one birth). Results are reported as ORs with associated two-tailed 95\% CIs.

The primary analysis was done without replacement of missing values, which was considered justified because attrition occurred only for covariates and was less than $2 \%$. However, a sensitivity analysis was also done by using a generalised estimating equations model with missing data filled in through multiple imputation to test the robustness of the findings of the primary analysis.

A two-tailed $p$ value of 0.05 or less was used to define statistical significance. By setting type- 2 error at the same severity level as the type- 1 error, multiplicity generated by the 48 comparisons was handled by controlling the false discovery rate as an alternative to the classic Bonferronitype adjustments. ${ }^{22}$ This adjustment was done by the Benjamini and Hochberg adaptive linear step-up approach. ${ }^{23}$ All statistical calculations were done with SAS (version 9.4).

\section{Role of the funding source}

The funders of the study had no role in study design, data collection, data analysis, data interpretation, or writing of the report. The data are the property of the EURAP study group and the authors had full access to all data. The Central Project Commission of EURAP had the final responsibility for the decision to submit for publication.

\section{Results}

Of the 21875 pregnancies registered in the database from June 20, 1999, to May 20, 2016, 9089 pregnancies were identified prospectively in women who had been exposed to antiepileptic drug monotherapy and had complete follow-up data up to 1 year. Of these pregnancies, 7555 met eligibility criteria (figure). 237 (3\%) of the 7555 pregnancies were enrolled through the UK and Ireland Epilepsy and Pregnancy Register, 419 (6\%) from the Australian Pregnancy Register, and 226 (3\%) from the Kerala Registry of Epilepsy and Pregnancy. Of the 7555 eligible pregnancies, 2514 (33\%) were exposed to lamotrigine, 1957 (26\%) to carbamazepine, 1381 (18\%) to valproate, $599(8 \%)$ to levetiracetam, $333(4 \%)$ to oxcarbazepine, $294(4 \%)$ to phenobarbital, 152 (2\%) to topiramate, and $125(2 \%)$ to phenytoin. The remaining $200(3 \%)$ pregnancies were associated with 13 other antiepileptic drug monotherapy exposures (ranging from one to 45 pregnancies in each group). Table 1 summarises the demographics of the 7355 pregnancies exposed to the eight most common monotherapies. These pregnancies resulted in 7275 live-born offspring, 43 perinatal deaths, and 37 elective terminations for fetal abnormalities.

810 women contributed two pregnancies, 71 women to three pregnancies, two women to four pregnancies, and 
one woman to five pregnancies. There were 106 twin pregnancies and one triplet pregnancy. Each twin or triplet was considered a separate pregnancy. Therefore, the 7355 pregnancies occurred in 6393 women.

The patient's physicians submitted reports on potential abnormal fetal outcomes in 752 pregnancies; 383 (5\%) of the total 7355 pregnancies were classified as major congenital malformations by the committee. The prevalence of major congenital malformations was $142(10 \cdot 3 \%)$ of 1381 pregnancies exposed to valproate, $19(6 \cdot 5 \%)$ of 294 to phenobarbital, eight $(6 \cdot 4 \%)$ of 125 to phenytoin, $107(5.5 \%)$ of 1957 to carbamazepine, six $(3.9 \%)$ of 152 to topiramate, ten $(3.0 \%)$ of 333 to oxcarbazepine, $74(2.9 \%)$ of 2514 to lamotrigine, and $17(2 \cdot 8 \%)$ of 599 to levetiracetam (table 2$)$. Outcomes of pregnancies exposed to the 13 less frequently used antiepileptic drugs, which were not included in further analyses, are presented in the appendix. A dose dependency in the prevalence of major congenital malformations was identified for carbamazepine, lamotrigine, phenobarbital, and valproate (table 3).

Table 4 shows the comparisons between the treatment associated with the lowest frequency of major congenital malformations (lamotrigine $\leq 325 \mathrm{mg} /$ day) and other antiepileptic drug treatments, within-drug comparisons of different dose categories, 15 other between-drug comparisons, and non-drug related comparisons. Compared with lamotrigine of $325 \mathrm{mg}$ /day or less, the odds of major congenital malformations was significantly higher for all doses of carbamazepine and valproate, and for phenobarbital at doses of more than $80 \mathrm{mg} /$ day. The low dose category of valproate $(\leq 650 \mathrm{mg} /$ day) was associated with increased odds compared with levetiracetam, whereas high-dose carbamazepine (>700 mg/day) was associated with increased odds compared with levetiracetam and oxcarbazepine. Low-dose valproate ( $\leq 650 \mathrm{mg} /$ day) was not associated with increased odds compared with high-dose lamotrigine (>325 mg/day). Increased odds were also associated with parental history of major congenital malformations and with pregnancies from southeast Asia compared with those from Europe (table 4). Appropriate versus inappropriate or no use of folic acid supplementation was not associated with a reduced odds of major congenital malformations (table 4). No reduction in odds with appropriate use of folic acid was seen when results were analysed by different antiepileptic drugs or for different types of major congenital malformations (data not shown). The results of the sensitivity analysis are reported in the appendix and confirm the robustness of our primary analyses.

The different types of major congenital malformations observed in the 383 offspring are listed in the appendix. With respect to broad categories of malformations, cardiac congenital malformations were the most common, followed by hypospadias, multiple major congenital malformations, renal defects, and neural tube defects (table 5). An ultrasound examination was done in 365 of the
383 pregnancies ending with a major congenital malformation, revealing the malformation in 76 of these cases.

\section{Discussion}

In this large cohort study, we ascertained the comparative risk of major congenital malformations for eight commonly used antiepileptic drugs. We did multivariable analysis of 36 direct comparisons between treatment modalities by using, as a measure of exposure, the dose taken at time of conception, which is the dose that physicians can best relate to for treatment decisions before planned pregnancies. We are aware that dose at conception is only an approximate measure of exposure, not least because changes in plasma antiepileptic drug concentrations can occur during pregnancy, including the first trimester. In line with our earlier analysis based on much smaller cohorts, ${ }^{5}$ we identified a dose dependency of the major congenital malformation risk for lamotrigine, carbamazepine, valproate, and phenobarbital. Additionally, we found that even at the lowest dose category ( $\leq 650 \mathrm{mg} /$ day), valproate was associated with significantly greater risk than most other treatments, with the exception of high-dose categories of carbamazepine and lamotrigine. The lowest risk was found with low-dose lamotrigine ( $\leq 325 \mathrm{mg} /$ day). This study provides data on the largest published cohorts with levetiracetam $(n=599)$ and oxcarbazepine $(n=333)$ monotherapy exposure, for which the risk was not dose dependent and overall was comparable to that of low-dose lamotrigine and lower than high-dose carbamazepine. Our data for phenytoin and topiramate, for which no dose dependency was found, need to be interpreted more cautiously because of the comparatively fewer exposures.

Parental history of major congenital malformations was associated with an almost three-times increase in

\begin{tabular}{|c|c|c|}
\hline & $\begin{array}{l}\text { Odds ratio } \\
(95 \% \mathrm{Cl})\end{array}$ & $\begin{array}{l}\text { Adjusted } \\
\text { p value } †\end{array}$ \\
\hline \multicolumn{3}{|l|}{ (Continued from previous page) } \\
\hline \multicolumn{3}{|l|}{ Non-drug covariates } \\
\hline Parental history of major congenital malformations & $2.98(1.52-5 \cdot 87)$ & 0.0023 \\
\hline Southeast Asia vs Europe & $2.43(1.48-3.99)$ & 0.0009 \\
\hline Americas vs Europe & $1.51(0.62-3 \cdot 70)$ & 0.3673 \\
\hline Sex (boys vs girls) & $1.33(1.07-1.67)$ & 0.0142 \\
\hline Folic acid (appropriate use vs no use or inappropriate use) & $1.26(0.99-1.60)$ & 0.0579 \\
\hline Maternal age at conception $\ddagger$ & $1.01(0.98-1.03)$ & 0.5166 \\
\hline Western Pacific vs Europe & $0.94(0.63-1.40)$ & 0.7440 \\
\hline Generalised tonic-clonic seizures during first trimester & $0.91(0.58-1.43)$ & 0.6890 \\
\hline Undetermined or unclassifiable vs localisation-related epilepsy & $0.76(0.51-1.14)$ & 0.1893 \\
\hline Parity 1 vs 0 & $0.75(0.58-0.98)$ & 0.0329 \\
\hline Idiopathic generalised epilepsy vs Localisation-related epilepsy & $0.74(0.56-0.98)$ & 0.0383 \\
\hline Parity $\geq 2$ vs 0 & $0.72(0.46-1.13)$ & 0.1574 \\
\hline
\end{tabular}

Within each category of comparison, treatments and other variables are listed in decreasing order of odds, from highest to lowest odds. ${ }^{*} n=7355$. † Multiplicity generated by the 48 comparisons was handled by controlling false discovery rate. $\ddagger$ Continuous covariate.

Table 4: Results of multivariable analysis of major congenital malformations in the study cohort ${ }^{*}$ 


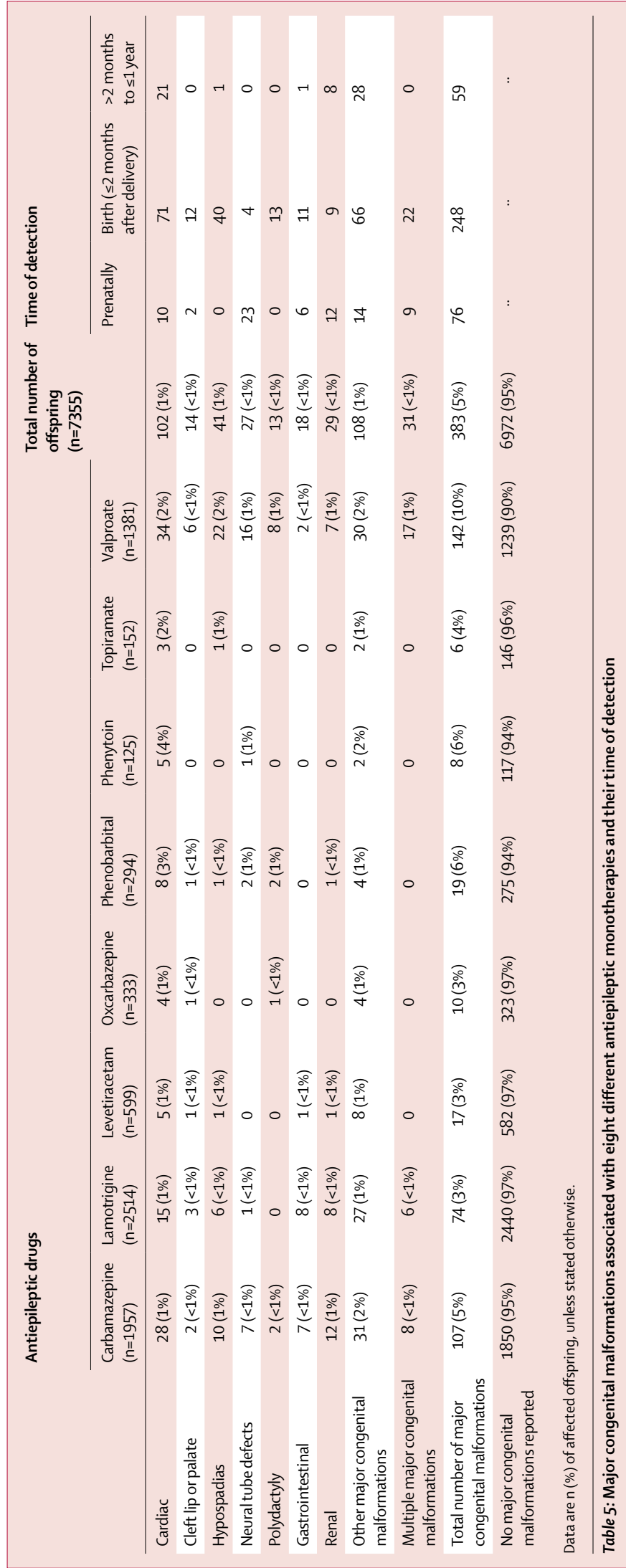

odds of major congenital malformations, suggesting a genetic predisposition to malformations or an individual susceptibility, or both, to the teratogenic effects of antiepileptic drugs. Earlier studies have found a high risk of major congenital malformations in the offspring of women who had multiple pregnancies associated with exposure to the same drug. ${ }^{24,25}$ Compared with inappropriate or no folate supplementation, appropriate folate supplementation was associated with a borderline (about 1-3-times) increase in the odds of major congenital malformations, although this finding was not significant. This finding might reflect a greater propensity to use folate in women considered to be at greater risk of an adverse pregnancy outcome and should not be considered as an argument against folate supplementation. The results, however, confirm the paucity of evidence for a protective effect of folate against major congenital malformations induced by antiepileptic drugs. ${ }^{26}$ Our analysis also revealed about a 1.3-times increase in odds of major congenital malformations in offspring of the male sex. The sex difference was no longer present when the sex-specific hypospadias was removed from the analysis (data not shown).

EURAP has a time window for identification of major congenital malformations extending to 1 year after birth, whereas other registries mostly limit their follow-up to birth or 2 months after birth., ${ }^{2,3}$ Of the 383 cases with major congenital malformations, $59(15 \%)$ cases were only detected after 2 months, which explains why the frequency of major congenital malformations is higher in EURAP than in studies with shorter follow-up. This extended duration of follow-up, together with differences in criteria of major congenital malformations, limits comparisons across registries ${ }^{27}$ and affects the validity of meta-analyses. ${ }^{1,19}$

It is concerning that only $20 \%$ of major congenital malformations were detected prenatally, despite the fact that $95 \%$ of the women had undergone an ultrasound examination, which even failed to detect four (15\%) of 27 neural tube defects. The detection rate is known to vary markedly depending on the type of major congenital malformation, examination modalities used, and examiner's expertise. In a systematic review, a second trimester ultrasound scan was found to detect spina bifida in $67 \%$ of offspring, cardiac defects in $17 \%$, gastrointestinal defects in $41 \%$, and renal malformations in $67 \% .^{28}$ The detection rates for cardiac defects, gastrointestinal defects, and renal malformations were lower in the EURAP registry than in this systematic review. In line with the observational nature of the registry, ultrasonography testing was not done according to a standardised protocol, but followed local standards in participating centres. Most of the pregnancies, however, had an examination in the second trimester.

EURAP and other prospective registries have previously reported frequencies of major congenital malformations after monotherapy exposure to the most commonly used antiepileptic drugs based on smaller cohorts..$^{2-5,18}$ The 
North American Antiepileptic Drug Pregnancy Registry reported a dose dependency in risk of major congenital malformations for valproate only, and found that in comparison with lamotrigine the risk was significantly greater for valproate (risk ratio $5 \cdot 1,95 \%$ CI 3.0-8.5), phenobarbital $(2 \cdot 9,1 \cdot 4-5 \cdot 8)$, and topiramate $(2 \cdot 2$, $1 \cdot 2-4 \cdot 0) .^{3}$ The UK and Ireland Epilepsy and Pregnancy Registers reported greater risk of major congenital malformations with valproate than with lamotrigine and carbamazepine, and found a dose dependency in the risk associated with valproate and carbamazepine. ${ }^{2}$ The Australian Pregnancy Register ${ }^{29}$ and the Kerala Registry of Epilepsy and Pregnancy, ${ }^{4}$ both collaborating with EURAP, have reported dose dependency in the risk of major congenital malformation with valproate. The comparatively low frequency of major congenital malformations with levetiracetam and oxcarbazepine found in our study is in line with reports from smaller cohorts. ${ }^{3,29,30}$ By contrast with some studies,,$^{1,319,31}$ we did not find a particularly high risk with topiramate. Our results on topiramate should, however, be interpreted cautiously because of the smaller size of the cohort exposed to this drug.

Our analysis provides a large number of direct comparisons between treatments, including risks associated with specific dose ranges, which can guide treatment choices for women of childbearing potential who require antiepileptic drug treatment.

The main strengths of our study are the large size of the cohort, the prospective recording of exposure to antiepileptic drugs and other potentially relevant risk factors before outcome is known, and the meticulous follow-up with strict criteria for classifying major congenital malformations. A further strength, and a challenge, is the international scope with inclusion of data from more than 40 countries worldwide. One limitation is that the registry is not population based, and women are enrolled by reporting physicians who might have a special interest and expertise. Such possible selection bias should, however, not affect the validity of the internal comparisons between treatments, which is the main objective of the study. A further limitation is the absence of a control group of pregnancies without exposure to antiepileptic drugs, but the relevant question for physicians treating women with a serious condition that involves pregnancy is rarely whether to treat or not, but rather which of the available treatments is the safest. However, a recent large meta-analysis reported an average risk of major congenital malformations of $2 \cdot 6 \%$ in children unexposed to antiepileptic drugs. ${ }^{1}$ Although it is difficult to compare data across studies, the frequency of major congenital malformations associated with lamotrigine, levetiracetam, and oxcarbazepine in the EURAP registry are in the same range as those reported in the meta-analysis for unexposed offspring. One might also consider the fact that exposure was defined by antiepileptic drug dose rather than plasma concentration of the antiepileptic drug as a limitation, because plasma concentration probably better reflects fetal exposure to the drug. However, standardised drug concentration sampling and measurement was not feasible in a large-scale international epidemiological study. Furthermore, there is no consensus on when samples should be drawn or how they should be analysed to best reflect fetal exposure during the critical time window for teratogenesis.

Because treatment allocation was not randomised, factors other than the type of antiepileptic drug used might affect outcome. For these reasons, we excluded pregnancies with exposure to other teratogenic drugs or with concurrent diseases known to increase the risk of adverse pregnancy outcomes. Information about other potential risk factors was collected prospectively and included in the multivariable analysis.

In conclusion, this study is the most comprehensive direct comparison of major congenital malformation risk with different antiepileptic drugs. Therefore, this study provides physicians with vital information when they consider different treatment alternatives for women that are of childbearing potential. Although our analysis was restricted to women with epilepsy, there is no reason why the data should not be applicable to fetal antiepileptic drug exposure regardless of indication.

\section{Contributors}

All authors contributed equally to the design of the study. EB did the statistical analysis. All authors contributed equally to the interpretation of the data. TT and DB drafted the first version of the manuscript, and all authors contributed equally to the input of the first draft and the finalisation of the submitted manuscript.

\section{Declaration of interests}

TT is an employee of Karolinska Institutet (Stockholm, Sweden) and is Associate Editor for the Epileptic Disorders journal. TT has also received speaker's honoraria to his institution from Eisai, UCB, Actavis, BMJ India, Livanova, and Sandoz; honoraria to his institution for advisory boards from UCB and Eisai; and research support from Stockholm County Council, CURE, GlaxoSmithKline, UCB, Eisai, Bial, and Novartis. DB has received speaker's honoraria from UCB and Sanofi. EB has received consultancy fees from Italfarmaco, Zambon, Polichem, Roche, and Sanofi-Aventis. JC has received research grants and speaker's honoraria from UCB Pharma, Eisai, GlaxoSmithKline, Sanofi Synthelabo, Pfizer, and Janssen-Cilag. DL has received research grants from Janssen-Cilag, GlaxoSmithKline, Pfizer, and the Netherlands Epilepsy Foundation. EP has received research funds from the European Union, the Italian Ministry of Health, the Italian Ministry for Education and University, and the Italian Medicines Agency; has received speaker's, consultancy fees, or research grants from Eisai, Livanova, Medichem, Sanofi, Sandoz, and Sun Pharma; and has been on the safety or advisory boards of UCB Pharma and GW Pharma. AS has received consultancy or lecture fees for Eisai Denmark and UCB Nordic; and has received travel support from Eisai, Biogen, and UCB Nordic. SVT has received research support from the Indian Council of Medical Research, speaker's honorarium from BMJ India, and Royalty from Wiley Publishing House. FV declares no competing interests.

\section{Acknowledgments}

EURAP has received financial support from Bial, Eisai,

GlaxoSmithKline, Novartis, UCB, Janssen-Cilag, Pfizer, and

Sanofi-Aventis. EURAP have continued to receive financial support after 2010 from Bial, Eisai, GlaxoSmithKline, Novartis, and UCB. Further financial support has been provided from the Netherlands Epilepsy Foundation (grant 03-18 [Lindhout]) and the Regional Agreement on Medical Training and Clinical Research (ALF) between Stockholm County Council and Karolinska Institute (Tomson). The Australian 
Pregnancy Register has received funding from Sanofi/Genzyme, UCB, Janssen, Sci-Gen, Eisai, the Australian National Health and Medical Research Council, Epilepsy Society of Australia, Royal Melbourne Hospital Medical Research Foundation, and Epilepsy Action.

\section{References}

1 Veroniki AA, Cogo E, Rios P, et al. Comparative safety of anti-epileptic drugs during pregnancy: a systematic review and network meta-analysis of congenital malformations and prenatal outcomes. BMC Med 2017; 15: 95.

2 Campbell E, Kennedy F, Russell A, et al. Malformation risks of antiepileptic drug monotherapies in pregnancy: updated results from the UK and Ireland Epilepsy and Pregnancy Registers. J Neurol Neurosurg Psychiatry 2014; 85: 1029-34.

3 Hernandez-Diaz S, Smith CR, Shen A, et al. Comparative safety of antiepileptic drugs during pregnancy. Neurology 2012; 78: 1692-99.

4 Thomas SV, Jose M, Divakaran S, Sankara Sarma P. Malformation risk of antiepileptic drug exposure during pregnancy in women with epilepsy: results from a pregnancy registry in South India. Epilepsia 2017; 58: 274-81.

5 Tomson T, Battino D, Bonizzoni E, et al. Dose-dependent risk of malformations with antiepileptic drugs: an analysis of data from the EURAP epilepsy and pregnancy registry. Lancet Neurol 2011; 10: 609-17

6 Tomson T, Battino D, Perucca E. Valproic acid after five decades of use in epilepsy: time to reconsider the indications of a time-honoured drug. Lancet Neurol 2016; 15: 210-18.

7 Vajda FJ, O’Brien TJ, Graham JE, Lander CM, Eadie MJ. Dose dependence of fetal malformations associated with valproate. Neurology 2013; 81: 999-1003.

8 Baker GA, Bromley RL, Briggs M, et al. IQ at 6 years after in utero exposure to antiepileptic drugs: a controlled cohort study. Neurology 2015; 84: 382-90.

9 Meador KJ, Baker GA, Browning N, et al. Cognitive function at 3 years of age after fetal exposure to antiepileptic drugs. N Engl J Med 2009; 360: 1597-605.

10 Meador KJ, Baker GA, Browning N, et al. Fetal antiepileptic drug exposure and cognitive outcomes at age 6 years (NEAD study): a prospective observational study. Lancet Neurol 2013; 12: 244-52.

11 Christensen J, Gronborg TK, Sorensen MJ, et al. Prenatal valproate exposure and risk of autism spectrum disorders and childhood autism. JAMA 2013; 309: 1696-703.

12 US Food Drug Administraion. FDA drug safety communication: valproate anti-seizure products contraindicated for migraine prevention in pregnant women due to decreased IQ scores in exposed children. June 5, 2013. https://www.fda.gov/Drugs/ DrugSafety/ucm350684.htm (accessed Sept 12, 2017)

13 European Medicines Agency. Assessment report: procedure under article 31 of directive 2001/83/EC resulting from pharmacovigilance data. Oct 9, 2014. http://www.ema.europa.eu/docs/en_GB/ document_library/Referrals_document/Valproate_and_related_ substances_31/Recommendation_provided_by_Pharmacovigilance Risk_Assessment_Committee/WC500177352.pdf (accessed April 10, 2015).

14 Wen X, Meador KJ, Hartzema A. Antiepileptic drug use by pregnant women enrolled in Florida Medicaid. Neurology 2015; 84: 944-50.
15 Murphy S, Bennett K, Doherty CP. Prescribing trends for sodium valproate in Ireland. Seizure 2016; 36: 44-48.

16 Tomson T, Marson A, Boon P, et al. Valproate in the treatment of epilepsy in girls and women of childbearing potential. Epilepsia 2015; 56: 1006-19.

17 Bobo WV, Davis RL, Toh S, et al. Trends in the use of antiepileptic drugs among pregnant women in the US, 2001-2007: a medication exposure in pregnancy risk evaluation program study. Paediatr Perinat Epidemiol 2012; 26: 578-88.

18 Vajda FJ, O'Brien TJ, Lander CM, Graham J, Eadie MJ. The teratogenicity of the newer antiepileptic drugs-an update. Acta Neurol Scand 2014; 130: 234-38.

19 de Jong J, Garne E, de Jong-van den Berg LT, Wang H. The risk of specific congenital anomalies in relation to newer antiepileptic drugs: a literature review. Drugs Real World Outcomes 2016; 3: 131-43.

20 Proposal for revised classification of epilepsies and epileptic syndromes. Commission on Classification and Terminology of the International League against Epilepsy. Epilepsia 1989; 30: 389-99.

21 EUROCAT. EUROCAT Guide 1.3 and reference documents: instructions for the registration and surveillance of congenital anomalies. Newtownabbey: EUROCAT Central Registry, 2005.

22 Glickman ME, Rao SR, Schultz MR. False discovery rate control is a recommended alternative to Bonferroni-type adjustments in health studies. J Clin Epidemiol 2014; 67: 850-57.

23 Benjamini Y, Hochberg Y. Controlling the false discovery rate: a practical and powerful approach to multiple testing. J R Stat Soc Series B Stat Methodol 1995; 57: 289-300.

24 Vajda FJ, O’Brien TJ, Lander CM, Graham J, Roten A, Eadie MJ. Teratogenesis in repeated pregnancies in antiepileptic drug-treated women. Epilepsia 2013; 54: 181-86.

25 Campbell E, Devenney E, Morrow J, et al. Recurrence risk of congenital malformations in infants exposed to antiepileptic drugs in utero. Epilepsia 2013; 54: 165-71.

26 Morrow JI, Hunt SJ, Russell AJ, et al. Folic acid use and major congenital malformations in offspring of women with epilepsy: a prospective study from the UK Epilepsy and Pregnancy Register. J Neurol Neurosurg Psychiatry 2009; 80: 506-11.

27 Tomson T, Battino D, Craig J, et al. Pregnancy registries: differences, similarities, and possible harmonization. Epilepsia 2010; 51: 909-15.

28 Bricker L, Garcia J, Henderson J, et al. Ultrasound screening in pregnancy: a systematic review of the clinical effectiveness, cost-effectiveness and women's views. Health Technol Assess 2000; 4: 1-193.

29 Vajda FJ, O'Brien T, Lander C, Graham J, Eadie M. The efficacy of the newer antiepileptic drugs in controlling seizures in pregnancy. Epilepsia 2014; 55: 1229-34.

30 Mawhinney E, Craig J, Morrow J, et al. Levetiracetam in pregnancy: results from the UK and Ireland epilepsy and pregnancy registers. Neurology 2013; 80: 400-05.

31 Hunt S, Russell A, Smithson WH, et al. Topiramate in pregnancy: preliminary experience from the UK Epilepsy and Pregnancy Register. Neurology 2008; 71: 272-76. 